\title{
A Review on Classification of Fingerprint Images
}

\author{
Mridula, Priyanka \\ Electronics and communication Engg Deptt, Deenbandhu Chhotu Ram University of Science and Technology, \\ Murthal, India
}

\begin{abstract}
Fingerprint is most widely used in individual identification especially in civilian applications, forensic laboratories etc. due to large bio-invariant characteristics of human fingerprints. A search over all the records in a data base takes a very long time and deteriorates accuracy as database is very large. To avoid this inconvenience, automatic fingerprint classification method is employed that does not require any user's interaction, reduce the search space and time and also increases accuracy of identifications. Classifying fingerprint images is a very difficult pattern recognition problem, due to the small interclass variability, the large intraclass variability, and the presence of noise and the ambiguous properties of fingerprints. In this paper we discuss about fingerprint classification and also review existing methods that have been applied for the classification problem.
\end{abstract}

Keywords: Minutiae, Ridges, Fingerprint Classification, Core Point, Delta Point

\section{Introduction}

Biometric systems operate on behavioural and physiological characteristics to identify a person. Behavioural biometric parameters include signature, voice, keystroke etc., that may change with time and physiological characteristics include face, fingerprint, palm print, iris recognition etc, which remain same throughout the life of an individual. Automatic Fingerprint Identification System (AFIS) is most widely used biometric due to large number of bio invariant characteristics of a human fingerprint.

Each fingerprint in this world is unique and each person of this world has a unique fingerprint with permanent unique characteristics over it. As database is very large, so to efficiently match fingerprints in this large database, classification of fingerprint is necessary. Fingerprint classification refers to assigning a given fingerprint image to one of the existing classes already recognized in the literature. This method is used to classifying a fingerprint to a class in a consistent and reliable way. A common approach is to partitioning the fingerprint database into a number of classes. A fingerprint to be identified is then required to be compared only to the fingerprints in a single bin of database based on its class. Fingerprint classification not only reduces comparisons of fingerprint but also increases overall efficiency of the system [1].

Suppose Inputs are the fingerprint impressions from 15 fingers of an individual. If size of the database is $\mathrm{N}$ and $\mathrm{C}$ is the number of classes, then search space without classification has $\mathrm{N}^{15}$ members while with classification search space will have only (N/C) ${ }^{15}$ members. [2]

The information carrying features in a fingerprint are the ridges and valleys. The Minutiae provides the details of ridge - valley structures like ridge endings and bifurcations [3] as shown in Fig. 1. Good quality fingerprint contains 40-100 minutiae. Poor fingerprint images leads to following problems: 1) some spurious minutiae can be created. 2) A large number of genuine minutiae can be ignored. 3) Errors in localisation.

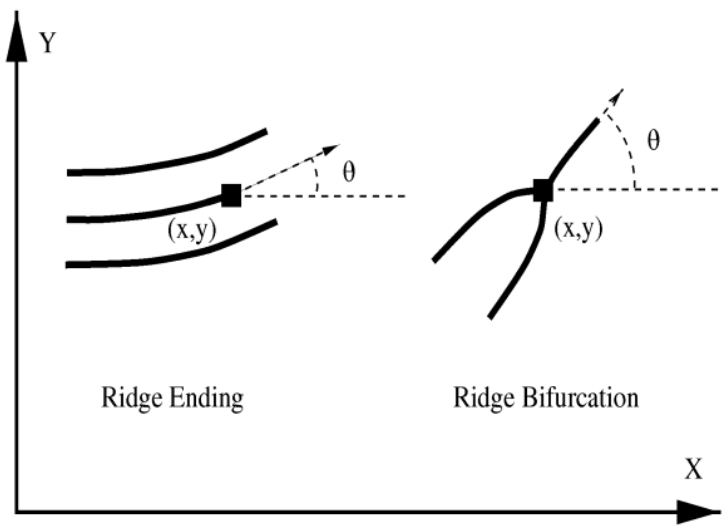

(a) 


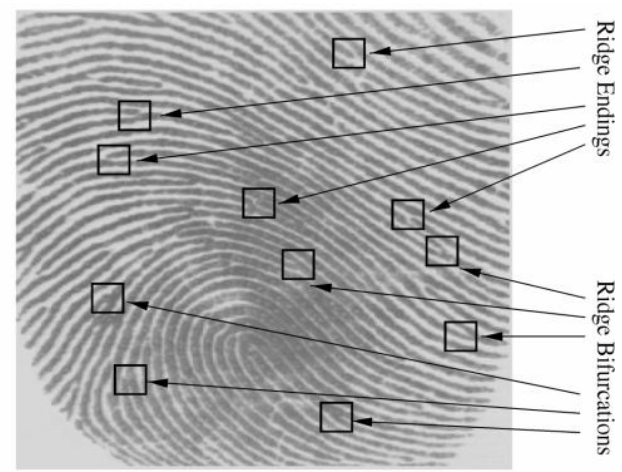

(b)

Fig.1 examples of minutiae: (a) a minutiae can be characterized by its position and orientation (b) minutiae overlaid on fingerprint image.

\section{Classification Of Fingerprint Images}

Henry's Classification [5] scheme divides a fingerprint structure into three major classes or patterns namely Arch, Loop and Whorl. These classes are further divided by researchers into five subclasses: Plain arch, tented arch, Left loop, Right loop and Whorl which can be briefly described below:

\subsection{Loops}

Loops occur in 60-65\% of fingerprint patterns encountered. In this, one or more of the ridges enters on either side of fingerprint impression, re-curve and terminates in the direction from where the ridges entered. Each loop pattern has one delta and one core,

There are two types of loops:-

- Ulnar loop - Loop opening towards little finger is called ulnar loop. Ulnar loops are named after ulna bone, a bone in the forearm. The ulna is on the same side as the little finger and it runs in the direction towards the little finger as shown in fig. 2.1(a)

- Radial loop - Loop opening toward thumb is called radial loop. Radial loops are named after radius where radius is the lateral bone, a bone in the forearm. The radial is on the same side as the thumb and it runs in direction towards thumb as shown in fig. 2.1(b). These are not very common and sometimes found on index finger.

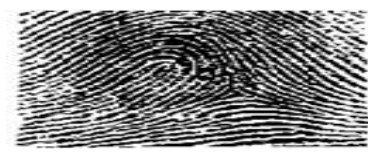

(a)

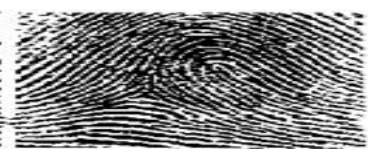

(b)

Fig.2.1 (a) ulnar loop (b) radial loop

\subsection{Whorls}

A whorl is roughly circular i.e. consist of one or more free recurving ridge lines going all the way around and it is more complex. It is always characterized by at least two points of deltas and must have type lines [4]. Whorls occur in 30-35 percent of fingerprint patterns encountered. When the line of the fingerprint disc is placed on the two points of delta, it will bisect at least one of the ridges belonging to the core group [5].

Four major types of whorls are: plain whorl, central pocket whorl, double loop whorl and accidental whorl.

(a)Plain whorls must have at least one ridge that makes a complete circuit, and an imaginary line from one delta to the other must touch a whorl ridge [4] as shown in fig. 2.2(a).

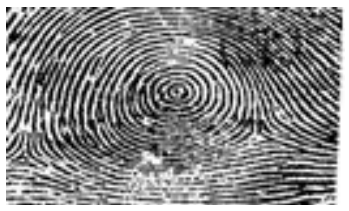

Fig. 2.2(a) plain whorl 
(b)Central pocket whorls must have at least one ridge that makes a complete circuit and an imaginary line from one delta to the other that cannot touch a whorl ridge [4] as shown below:

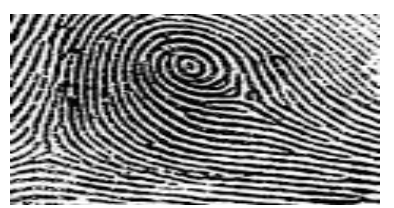

Fig. 2.2(b) central pocket whorl

(c)Double loop whorls consist of formation of two separate and distinct loop with two separate and distinct shoulders for each core, two deltas and one or more ridges which make a complete circuit. In this, at least one re-curving ridge, within the pattern area, is cut or touched when an imaginary line is drawn [5] (fig. 2.2(c)).

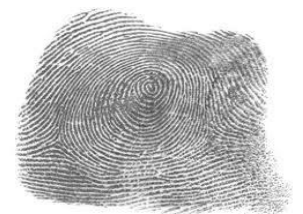

Fig. 2.2(c) double loop whorls

(d)Any other type not in the three categories are called accidentals whorl as shown in fig. 2.2(d).

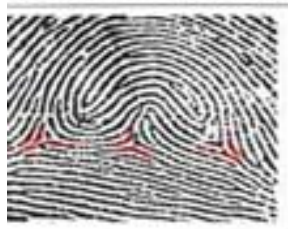

Fig. 2.2(d) Accidental whorls

\subsection{Arches}

In arch pattern, ridges run from one side to other with no backward turn. Arches do not have type line, delta or core. Only 5 percent of population has fingerprints of arch type pattern [4].

There are two types of arches:

Plain arch have an even flow of ridges from one side of pattern to the other (fig. 2.3(a)). There is no

"significant up thrusts". The ridges enter on one side of the impression, and flow out from the other side with a rise or wave in the centre [5].

Tented arch has an angle, a significant upthrust, or some basic characteristics of loop [5]. They don't have an easy flow as that of plain arch as shown in fig. 2.3(b).

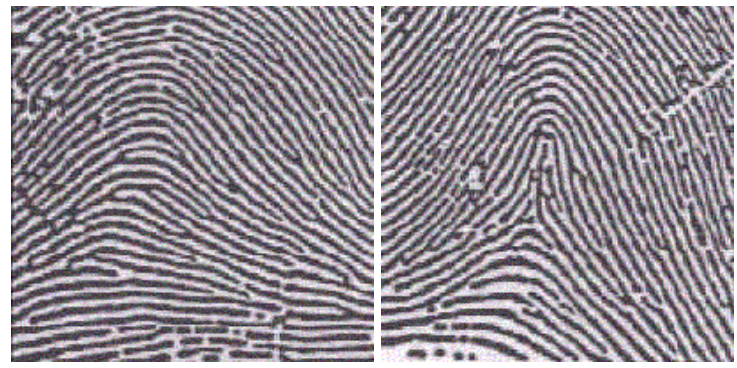

Fig. 2.3 (a) Plain arch $\quad$ (b) tented arch

\section{Different Methods Of Fingerprint Classification}

\subsection{Rule Based Approach}

The rule based approach codifies the human expert knowledge of manual classification such as the singular point extraction and geometrical shape of ridge lines [6].This is the most straightforward method than the others. This method relies on the acquired number and positions of the extracted singular points to classify fingerprints [7].

Repetitive regularization is done until a valid number of singular points are detected so that the noise can be reduced and also improves the accuracy. 


\section{Some Problems associated with Rule Based Approach are:}

-Although a rule based approach is simple and worked well in high image quality fingerprints but in poor quality fingerprints, robust and consistent detection of singular point is a difficult task. So, this approach is in general sensitive to noise [6].

-May work well on nail to nail finger print impressions scanned from cards but are not suitable for live scan fingerprint images because delta points are missing in these types of images[8].

\subsection{Syntactic Approach}

A Syntactic method represents a fingerprint by the features extracted from the ridge flow or orientation field. This method adopted basic ridge patterns, analysis of ridge shape and sequence of ridge shapes. In this approach, a grammar is defined for each fingerprint class. Classification is performed by determining which grammar most likely generates the feature extracted from a query fingerprint [6].

\section{Problem associated with syntactic approach is:}

This method tends to be robust in the presence of image noise but often require very complex grammar to struggle against the large intraclass and small interclass variations. Complex grammars often result in unstable classification.

\subsection{Structural Approach}

The structural approach organizes low level features into higher level structure. There are two types of structural approaches:

One of the approaches is to partition the orientation field into connected regions and relational graphs of these regions are employed to classify the fingerprint images. As a robust and consistent partition of orientation field is not an easy task then a template based matching is developed to guide the partitioning $[6,7]$.

Another approach converts the two dimensional fingerprint structures into one dimensional sequence. A set of horizontal lines across the fingerprints is used to extract some features that capture information about the local orientation and ridge structure and thus has higher discrimination power than local orientation alone[6].

Since structural approaches depend upon global structure information, they can work on noisy fingerprint images and also able to deal with those fingerprints in which singular points are not available.

\subsection{Statistical Approach}

The Statistical (STA) approach extract a fix size numerical feature vector from a fingerprint and the construction of feature vector is based on orientation field or the response of Gabor filters. [6] The features extracted from different images shows different discriminating powers. Some weighting schemes are developed to put higher weight in more discriminative regions of fingerprint images. Then some transform can be applied that can reduces the dimensionality of feature vector.

\subsection{Neural Network Approach}

The Neural Network (NN) approach is based on multilayer perceptron and the elements of orientation image as input. It consists of several multilayer perceptrons each of which is trained to recognize different classes of fingerprints. In this, the location of all the singular points are used together for the training of two disjoint neural networks and then its output is passed to third one which produces the final classification. [9] These approaches require lots of training data to yield the classifier, and sufficient data should be obtained to yield a more effective classifier.

\subsection{Multiple Classifier Approach}

Different parameters potentially offer extra information about the patterns to be classified, which may be exploited to improve performance of algorithm. It uses orientation field to detect core and delta points. Stage classification is done, firstly $\mathrm{K}$ nearest neighbour to find most likely classes and secondly neural network for further classification. It has used core and delta points and ridge flow as feature vector. Using singular point, ridge is traced in opposite directions to find the turn number. It then uses rules for classification [10].

\section{Relevant Terms For Classification}

\subsection{Ridges}

A ridge is a curved line in a finger. Some ridges may be continuous and some may be terminated at any point. When the ridges end at a point then these end points are called ridge endings. When two ridges meet at a point, then it is called ridge bifurcation. These ridge endings and bifurcation are called minutiae. 


\subsection{Core points}

Core point refers to centre area of fingerprint. In a whorl pattern, it is found in the middle of the spiral and in a loop pattern, it is found in the top region of the innermost loop. Basically, it is a U-turn in the ridge pattern.

\subsection{Delta point}

The delta is also known as tri- radiate ridge. It is a triangular shaped pattern where different fingerprint ridges meet. The delta point is a pattern of a fingerprint that resembles the Greek letter 'delta'. It is nearest to the point of divergence of two type line.

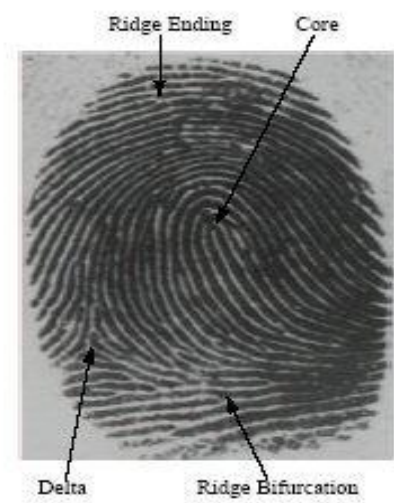

\section{Advantages Of Fingerprint Classification}

- Very well accepted in legal community among law enforcement and general public.

- Having long history in forensic science which gives it weight and credence that are not available to newer identification system.

- Provide high accuracy and reliability since two people on the earth can never have similar fingerprint.

- Reduces the size of database memory as fingerprint from someone can be matched only to that class from which it belongs.

- Easy to use and economical

- Most developed and standardized method

\section{Drawbacks Of Fingerprint Classification}

- If fingerprint evidence is not collected, stored and handled properly, then it may results in false identification.

- Lack of robustness in case of poor quality images.

- Reduced accuracy in the presence of noise.

\section{Conclusion And Research Scope}

Fingerprint classification approach inspired many researchers in this area. It provides the effective way of fingerprint verification method with reduced time and improved accuracy. There are various techniques have been applied for several years for classification of fingerprint images. The future work might be use combination of features extracted in existing techniques that provides more accuracy. So we tend to study more details in this area so that we will give better insights on how to improve quality of fingerprints in preprocessing steps.

\section{References}

[1]. Ahmad, F. ; Fac. of Computer Sci. \& Inf. Syst., Univ. Teknol. Malaysia, Mohammad, D.“A review on fingerprint classification technique" comp technology and development, vol.2, pp-411-415(2009)

[2]. 22. Sarat C. Dass "Classification of Fingerprints", Department of statistics and Probability (October 2010)

[3]. Yifei Wan, and Anil Jain, "Fingerprint image enhancement: algorithm and performance evaluation", IEEE transactions on pattern analysis and machine intelligence, vol. 20, no. 8, August 1998

[4]. Hong L., Jain A.K. 'Classification of Fingerprint Images'.

[5]. Proceedings of 11th Scandividian Conference on Image Analysis,(1999).

[6]. Fingerprint Patterns (based on Henry classification system) http://ridgesandfurrows.homestead.com/fingerprint_patterns.html

[7]. Anil K. Jain, Stan Z.Li "Encyclopedia of Biometrics" volume $1 \mathrm{http} / /$ www.amazon.com/Encyclopedia-Biometrics-Anil-KJain/dp/0387730044

[8]. Jing-Ming Guo, Yun-Fu liu, Jla-Yu Chang, Jiann-Der Lee: "Fingerprint classification based on decision tree from singular points and orientation 
[9]. Fields": Expert system with applications, vol. 41, issue2, pp.-752-764(February 2014)

[10]. Alessandra Lumini, Dario Maio and Davide Maltoni, "Fingerprint Classification by Directional Image Partitioning" IEEE transactions on pattern analysis and machine intelligence, vol. 21, no. 5, pp. 402-421, 1999.

[11]. Davide Maltoni, Dario Maio, Anil K. Jain, Salil Prabhakar "Handbook of fingerprint recognition"

[12]. Dimple Parekh, Rekha Vig," Survey on Parameters of Fingerprint Classification Methods Based On Algorithmic Flow" Vol.2, No.3, August 2011 